\title{
ESTILOS DE APREDNIZAGEM EM FÓRUNS ONLINE: PERSPECTIVAS PEDAGÓGICAS INOVADORAS ${ }^{1}$
}

\author{
LEARNING STYLES FOR ONLINE FORUMS: \\ INNOVATIVE PEDAGOGICAL PERSPECTIVES
ESTILOS DE APRENDIZAJE PARA LOS FOROS EN LÍNEA: PERSPECTIVAS PEDAGOGICAS INNOVADORAS

\author{
Daniela Melaré Vieira Barros ${ }^{2}$ \\ Vanessa Matos dos Santos ${ }^{3}$
}

\begin{abstract}
RESUMO: Cada vez mais as inovações tecnológicas potencializam estratégias e recursos para o trabalho educativo. Do ponto de vista pedagógico, os desafios são maiores e, nesta perspectiva, busca-se na teoria dos estilos de aprendizagem a facilitação e a compreensão de alguns aspectos dos processos educativos da atualidade, quais sejam: a personalização através das características individuais, inclusão e o uso das tecnologias no cenário educativo. A reflexão aqui desenvolvida tem por objetivo apresentar diretrizes para potencializar o trabalho pedagógico com os fóruns de discussão em contextos online, ampliando o conceito e a função pedagógica destas ferramentas com a teoria dos estilos de aprendizagem. A metodologia qualitativa utilizada no estudo é apresentada com base nas reflexões e experiências realizadas em contextos de ensino superior online. O estudo está fundamentado em um projeto maior, atualmente em desenvolvimento sobre a Didática Online. Para tanto, os fundamentos teóricos ampliam as discussões sobre dois casos práticos apresentados como exemplos que possibilitaram resultados que dialogam com as questões de pesquisa levantadas, em específico: como utilizar as tecnologias nas estratégias pedagógicas dos fóruns online a partir dos estilos de aprendizagem?
\end{abstract}

Palavras-chave: fórum online, estilos de aprendizagem, tecnologias digitais, estratégias pedagógicas

ABSTRACT: Technological innovations leverage strategies and resources for educational work are growing. From a pedagogical point of view, the challenges are greater and, in this perspective, the learning styles theory seeks to facilitate and understand some aspects of today's educational processes, namely: personalization through individual characteristics, inclusion and the use of technologies in the educational scenario. The purpose of the reflection developed

\footnotetext{
${ }^{1}$ Submetido em: 16/10/2017 - Aceito em: 08/06/2017 - Publicado em: 28/02/2018.

${ }^{2}$ Universidade Aberta, Centro de Estudos Interdisciplinares do Século XX - Coimbra, LE@D Laboratório de Educação e Ensino a Distância, UAb - Lisboa

${ }^{3}$ Universidade Federal de Uberlândia - Brasil

\begin{tabular}{c|c|c|c|c|c|c}
\hline (C) Redoc & Rio de Janeiro & v.2 & n.1 & p. 14 & Jan/Abr. 2018 & ISSN 2594-9004 \\
\hline
\end{tabular}
}




\section{Revista Docência e Cibercultura}

here is to present guidelines for enhancing pedagogical work with discussion forums in online contexts, expanding the concept and pedagogical function of these tools with the theory of learning styles. The qualitative methodology used in the study is presented based on the reflections and experiences carried out in online higher education contexts. The study is based on a larger project, currently under development on Didática Online. In order to do so, the theoretical foundations broaden the discussions on two practical cases presented as examples that allowed results that dialogue with the research questions raised, specifically: how to use the technologies in the pedagogical strategies of the online forums from the learning styles?

Keywords: Online forum, learning styles, digital technologies, pedagogical strategies

RESUMEN: Las estrategias de innovación tecnológica y los recursos para el trabajo educativo están creciendo. Desde un punto de vista pedagógico, los desafíos son mayores y, en esta perspectiva, la teoría de estilos de aprendizaje busca facilitar y comprender algunos aspectos de los procesos educativos actuales, a saber: personalización a través de características individuales, inclusión y uso de tecnologías en el escenario educativo . El propósito de la reflexión desarrollada aquí es presentar pautas para mejorar el trabajo pedagógico con foros de discusión en contextos en línea, ampliando el concepto y la función pedagógica de estas herramientas con la teoría de los estilos de aprendizaje. La metodología cualitativa utilizada en el estudio se presenta en base a las reflexiones y experiencias realizadas en contextos de educación superior en línea. El estudio se basa en un proyecto más grande, actualmente en desarrollo en Didática Online. Para ello, las bases teóricas amplían las discusiones sobre dos casos prácticos presentados como ejemplos que permitieron resultados que dialogaron con las preguntas de investigación planteadas, específicamente: ¿cómo utilizar las tecnologías en las estrategias pedagógicas de los foros en línea desde los estilos de aprendizaje?

Palabras clave: foro en línea, estilos de aprendizaje, tecnologías digitales, estrategias pedagógicas

\section{INTRODUÇÃO}

A base do processo educativo está na comunicação e nas trocas que se realizam entre os Sujeitos (FREIRE, 1983). Partindo deste ponto de vista, a comunicação deve ser não apenas o cimento, mas também o suporte de qualquer perspectiva educativa, quer seja em ambientes físicos ou online. Opta-se aqui pela terminologia "online", em detrimento de "a distância" em função da assunção de que as tecnologias digitais permitem hoje que o pressuposto da distância seja re-visto. É possível que o Sujeito esteja "presente", ainda que esteja localizado geograficamente distante (MOORE, 1997; PETERS, 2004).

Se em situações físicas a comunicação é importante, em contextos de aprendizagem online ela adquire importância potencializada. Para além das trocas de mensagens por meio de correio eletrônico (e-mail), os fóruns surgem como espaços essenciais na aprendizagem porque

n.1 1 p. 15




\section{Revista Docência e Cibercultura}

se traduzem em ferramentas que possibilitam a comunicação horizontal, desprovida de hierarquização. É sabido, no entanto, que os benefícios do uso dos fóruns na aprendizagem dos estudantes são conhecidos e comprovados em muitos estudos e produções científicas (MOKOENA, 2017; PETERS, 2004). Os estudos sobre o tema do fórum passam principalmente pela ferramenta web como instrumento inserido em ambientes de aprendizagem e como espaços de comunicação em geral. Como ator secundário nas investigações, o fóruns estão contextualizados como elementos dentro de um modelo pedagógico maior e amplo dos processos do online. A investigação em desenvolvimento está fundamentada em mecanismos da didática online para o trabalho em educação a distância no ensino superior.

Atualmente os fóruns ganharam uma outa dinamicidade com o movimento constante das interfaces online messenger e whatshapp. Esses espaços de comunicação - assíncrona ou síncrona - ganharam a responsabilidade dos fóruns de discussão tradicionalmente considerados como espaços únicos. Soma-se, a tudo isso, a contribuição de espaços de colaboração como as wikis (LOIDE ET AL., 2014; BIASUTTI, 2017). Considerando essa nova dinamicidade proporcionada pela tecnologia digital já numa perspectiva da qualidade e das novas tendências inovadoras dos dispositivos pedagógicos, o estudo aqui apresentado traz uma dinâmica integrada entre o fóruns como espaços mais dinâmicos e que podem ter estratégias pedagógicas de aprendizagem através da comunicação, partilha e participação (MELLO; BARROS, 2014a, 2014b). Importa, neste estudo, ampliar de forma qualitativa o potencial dessa ferramenta por meio do arcabolço dos estilos de aprendizagem (HONEY, ALONSO, GALLEGO, 2007), bem como os estilos de uso do espaço virtual (BARROS, 2008; 2009; 2013; 2015). Para tanto, a pergunta do estudo aqui apresentado é: como os fóruns online podem ser potencializados com estratégias didáticas e pedagógicas a partir da teoria de estilos de aprendizagem? Finalmente, a justificativa do estudo está em oferecer mais instrumentos e perspectivas do uso dos fóruns nos espaços de aprendizagem online. A lógica que guiou o estudo é o uso mais direcionado para a aprendizagem sistematizada mediante a qualidade da comunicação dinâmica e participativa. A estrutura deste documento segue a seguinte sequência: no primeiro momento apresenta, de forma sintetizada, algumas reflexões teóricas; no segundo momento os procedimentos metodológico do estudos, descrevendo em seguida os resultados e as conclusões dos mesmos.

\section{A COMUNICAÇÃO NOS FÓRUNS ONLINE}




\section{Revista Docência e Cibercultura}

Tradicionalmente, os fóruns online se traduzem em espaços de diálogo assíncrono entre estudantes e professor em plataformas de educação online. Estes espaços são abertos e permitem que os alunos troquem experiências e compartilhem dúvidas, pontos de vista, façam debates, etc. Os fóruns são, talvez, os principais espaços proporcionadores de comunicação horizontal nos ambientes virtuais de aprendizagem. Diferentemente de outros espaços, os fóruns praticamente planificam as diversas contribuições em uma discussão (DIAS, 2013). Ocorre, no entanto, que nem sempre estes espaços são utilizados em sua potencialidade (HENRI; LUNDGREN-CAYROL, 2001). Focados em uma visão meramente avaliativa, muitas vezes os Sujeitos (professores, alunos e tutores) tendem a sub-utilizar esta ferramenta. Muitos podem fazer um uso raso desta ferramenta por acreditarem que não se trata de um espaço avaliativo (GARRISON; CLEVELAND-INNES, 2005).

Entretanto, na vertente oposta, Titsworth (2017) destaca que os fóruns oline devem resgatar o aspecto interpessoal da comunicação. Mais que simplesmente rastros de passagem dos Sujeitos, é importante que estes espaços proporcionem agregação social, discussão, debate. A pesquisa conduzida por Mohamad e Shaharuddin (2014) demonstrou que, quando efetivamente utilizados, proporcionam o engajamento e o desenvolvimento da sensação de comunidade de aprendizagem notadamente entre os alunos. Este engajamento tem o potencial de manter a motivação do aluno de EAD por meio da troca e da partilha de ideias e opiniões. Trata-se de estar acompanhad e não apenas só no espaço virtual. O compartilhamento das dificuldades e também dos sucessos ajuda na visualização do Sujeito que está do outro lado do aparato tecnológico (MOKOENA, 2017), repondo a discussão feita por Linard em 1990 e resgatada em 2002: mais que aparatos tecnológicos, é preciso que exista a percepção do humano nos espaços de formação.

Neste aspecto, é importante ressaltar que os processos de trocas informacionais foram, com o advento das tecnologias digitais, ampliados e redimensionados na chamada cibercultura. Habermas (2003) menciona que um ato de comunicação constitui sempre uma ação efetuada por um sujeito, pois os intercâmbios do próprio pensamento obedecem também a uma lei de equilíbrio que constitui um agrupamento operatório, uma cooperação, mesmo que interna. A promoção dessas cooperações internas e externas pode ocorrer por diversas vias, como por exemplo os espaços virtuais e suas interfaces como os ambientes de aprendizagem. Essa 


\section{Revista Docência e Cibercultura}

cooperação interna e a externa contribui diretamente para um agir colaborativo em um ambiente de aprendizagem. É como um espaço presencial em que o diálogo se constitui e as pessoas se comunicam mediante proposições que surgem e vão se constituindo a partir da própria comunicação. Freire (1983) partiu do princípio de que a comunicação é a que transforma essencialmente os homens em sujeitos. Com esta base, formulou sua proposição fundamental de que a educação, como construção compartilhada de conhecimentos, constitui um processo de comunicação no diálogo, porque se gera através de relações igualitáriasentre os seres humanos e com o mundo.

Comunicação, por essa óptica, é algo muito maior, livre de materialidade. Ela se estabelece, entre outros aspectos, na relação com o outro, no princípio da alteridade, e é por essa razão que o Outro recebe especial atenção por parte de Ciro Marcondes Filho (2010). Também Buber (2001) faz uma importante reflexão a respeito do tu e do isso. A relação eu-tu é distinta da relação eu-isso. Enquanto a primeira pressupõe o encontro essencial do homem numa atitude de reciprocidade (posto que reconheço o tu), a segunda é calcada na atitude objetivante (o isso deve servir para ser investigado, transformado). A postura diz muito sobre o tipo de relação que se estabelece, posto que "tratar uma pessoa como objeto de estudo é vêla como 'isso'". Transformar o isso em tu pressupõe uma nova atitude, um novo comportamento, em que o eu se torna permeável ao outro (tu), pois, como destaca Buber (2001, p.56) "a alteridade essencial se instaura somente na relação EU-TU; no relacionamento EUISSO o outro não é encontrado como outro em sua alteridade". A comunicação pressupõe, desta forma, o reconhecimento do Sujeito, mas não apenas isso. É preciso romper a barreira que há em mim para acolher, hospedar o Outro que me choca (por ser tão diferente de mim) e que pode até mesmo me agredir dada a sua estranheza. É preciso abrir-se. Essa abertura, no entanto, não acontece sempre numa situação dialógica (como queria Buber); ela pode ocorrer pelo atrito, pelo radicalmente oposto, pela formação de ranhuras e fissuras na alma (SANTOS, 2017a). Nem sempre a opção por acolher o Outro ocorre sem atrito. Mas é justamente a formação dessas ranhuras que vai possibilitar uma guinada, uma transição, um salto qualitativo. Os fóruns online não devem gerar, nesta perspectiva, sempre concordância. Ademais, a discordância potencializa o debate, a pesquisa, a troca, o debate enfim. $\mathrm{O}$ fato de serem assíncronos permite, inclusive, que o Sujeito elabore e reelabore suasvisões para, então, colaborar com o debate numa perspectiva maior, autônoma e colaborativa. 


\section{Revista Docência e Cibercultura}

A aprendizagem colaborativa pode ser compreendida como um conjunto de métodos e técnicas de aprendizagem desenvolvidos em grupos, que envolvem elementos sociais e pessoais (competências) onde cada pessoa é responsável, por sua aprendizagem e de certa forma pela aprendizagem dos demais (PALLOFF; PRATT, 2004). Na aprendizagem colaborativa, a ênfase está na interação entre os participantes. Cabe ao(s) facilitador(es) ou moderador(es) propiciar situações de aprendizagem em que todos aprendam com todos (PALLOFF; PRATT, 2004). Aprendizagem colaborativarefere-se ao processo em que aprendizes trabalham em grupo, geralmente na produção de algo (um texto, um projeto, uma apresentação, um produto, etc.). Assim como ocorre com outras ferramentas educativas, os fóruns também exigem - do educador - diferentes competências (GRITSENKO, 2016; GERLOCK; MCBRIDE, 2013). A competência comunicativa pressupõe abertura para o diálogo e presença virtual (SANTOS, 2016; 2017b). Diferentemente do que ocorre em situações de educação face a face, nos espaços virtuais, os alunos percebem a presença do professor por meio de seus registros nos fóruns e demais ferramentas. O fórum é o espaço para a fala não-institucionalizada e, portanto, mais pessoal e que pode estabelecer um maior vínculo por meio da aproximação. Chang, Chen e Hsu (2011) explicam que a motivação do estudante depende da resposta rápida para suas perguntas. É importante que os professores destinem atividades (respostas) específicas para os estudantes, de forma a incentivar a colaboração, a partilha de responsabilidade.

\section{O APORTE DOS ESTILOS DE APRENDIZAGEM}

A terminologia "estilos" começou a ser difundida a partir do século XX, notadamente por pesquisadores da área de Psicologia e Educação (GARCÍA CUÉ, 2008). Inicialmente, os pesquisadores estavam interessados em descobrir como esses estilos se relacionavam com a aprendizagem de línguas e suas possíveis contribuições para o ensino. Segundo Goulão (2002), as pesquisas em Educação desde muito tempo tem demonstradó que diferentes pessoas tem diferentes formas e ritmos de aprendendizagem. Estas formas típicas de perceber e processar as informações são aquilo que, na literatura, se conhece por estilos de aprendizagem. Alguns psicólogos citados por Goulão (2002), como Riding e Rayner (1998 apud GOULÃO, 2002) e McLoughlin (1999 apud GOULÃO, 2002)), definiram os estilos de aprendizagem como uma tendência para abordar tarefas cognitivas através da utilização preferencial de uma estratégia

\begin{tabular}{l|l|l|} 
v.2 & n.1 & p. 19
\end{tabular}




\section{Revista Docência e Cibercultura}

ou de um conjunto de estratégias, isto é, da adoção, habitual e distinta, de um modelo para adquirir conhecimento. Isso significa que os estilos de aprendizagem afetam a forma de estar e de atuar dos Sujeitos em diferentes planos da vida. Afetam não só a forma como as pessoas aprendem, mas, também, como atuam em grupo, participam em atividades, se relacionam com os outros, resolvem problemas e trabalham (KOLB; SMITH,1996).

Os estilos de aprendizagem foram e são o foco de inúmeros estudos e, por essa razão, é possível encontrar diferentes formas de abordar o mesmo conceito, com o mesmo objetivo. Com base neste pressuposto de Grigorenko e Sternberg (apud GOULÃO, 2002) propõem três grandes e distintas perspectivas do conceito de estilo em Psicologia.

- Perspectiva centrada na cognição

- Perspectiva centrada na aprendizagem

- Perspectiva centrada na personalidade

Em 1976, David Kolb iniciou com a reflexão da repercussão dos estilos de aprender na vida adulta das pessoas explicando que cada indivíduo enfoca a aprendizagem de uma forma peculiar fruto da herança, experiências anteriores e exigências atuais do ambiente em que se move. Kolb identificou cinco forças que condicionam os estilos de aprendizagem: a de tipo psicológico, a especialidade de formação elegida, a carreira profissional, o trabalho atual e a capacidade de adaptação. Para Kolb (apud ALONSO, GALLEGO, HONEY, 2007) a aprendizagem é eficaz quando cumpre quatro etapas: experiência concreta, quando se faz algo; a observação reflexiva, quando se analisa e pondera; a conceptualização abstrata, quando se compara as teorias depois da análise; e, a experimentação ativa, que permite contrastar o resultado da aprendizagem com a realidade. Com base nessas quatro etapas, Kolb (apud ALONSO, GALLEGO, HONEY, 2007) destacou os estilos de aprendizagem e desenvolveu um questionário para sua identificação:

- acomodador: cujo ponto forte é a execução, a experimentação;

- divergente: cujo ponto forte é a imaginação, que confronta as situações desde múltiplas perspectivas; 


\section{Revista Docência e Cibercultura}

- assimilador: que se baseia na criação de modelos teóricos e cujo raciocínio indutivo é a sua ferramenta de trabalho; e,

- convergente: cujo ponto forte é a aplicação prática das ideias

Partindo das ideias e análises de Kolb (1981), Honey e Mumford (apud ALONSO, GALLEGO, HONEY, 2007) elaboraram um questionário a partir do qual se podem obter também quatro estilos diferentes de aprendizagem - Estilo ativista, Estilo reflexivo, Estilo teoricista e Estilo pragmático. Esta concepção de aplicação, no entanto, parte do local de trabalho, num aperspectiva organizacional.

Investigando essas teorias, Honey e Alonso, no ano de 1992, desenvolveram um estudo em que, na primeira parte tratava de centrar a problemática dos estilos de aprendizagem dentro das teorias gerais de aprendizagem, analisando criticamente o instrumento para a coleta de dados. A teoria dos estilos de aprendizagem trabalhada e refletida por esses investigadores está pensada mais na perspectiva da Educação e contempla também os aspectos sociais em que o indivíduo está inserido. Deta forma, ainda que existam diferentes concepções acerca dos estilos de aprendizagem, é importante destacar que, no escopo deste estudo, optou-se pela vertente espanhola amplamente desenvolvida por Alonso, Gallego e Honey (2007). Os estilos de aprendizagem de acordo com Alonso, Gallego e Honey (2007), com base nos estudos de Keefe (1998), são traços cognitivos, afetivos e fisiológicos, que servem como indicadores relativamente estáveis de como os alunos percebem, interagem e respondem a seus ambientes de aprendizagem. Existem quatro estilos definidos: o ativo, o reflexivo, o teórico e o pragmático.

- estilo ativo: valoriza dados da experiência, entusiasma-se com tarefas novas e é muito ágil. As pessoas em que o estilo ativo predomina, gostam de novas experiências, são de mente aberta, entusiasmadas por tarefas novas; são pessoas do aqui e do agora, que gostam de viver novas experiências. Suas características são: animador, improvisador, descobridor, que se arrisca, espontâneo.

- estilo reflexivo: atualiza dados, estuda, reflete e analisa. As pessoas deste estilo gostam de considerar a experiência e observá-la de diferentes perspectivas; reúnem dados, analisando-os 


\section{Revista Docência e Cibercultura}

com detalhamento antes de chegar a uma conclusão. Suas principais características são: ponderado, consciente, receptivo, analítico e exaustivo.

- estilo teórico: é lógico, estabelece teorias, princípios, modelos, busca a estrutura, sintetiza. Este estilo é mais frequente em pessoas que se adaptam e integram teses dentro de teorias lógicas e complexas. Profundos em seu sistema de pensamento e ao estabelecer princípios, teorias e modelos tendem a ser perfeccionistas integrando o que fazem em teorias coerentes. Buscam a racionalidade e objetividade se distanciado do subjetivo e do ambíguo; para eles se é lógico é bom.

- estilo pragmático: aplica a ideia e faz experimentos. Os pragmáticos são pessoas que aplicam na prática as ideias. Descobrem o aspecto positivo das novas ideias e aproveitam a primeira oportunidade para experimentá-las. Gostam de atuar rapidamente e com seguridade com aquelas ideias e projetos que os atraem. Tendem a ser impacientes quando existem pessoas que teorizam. Suas principais características são: experimentador, prático, direto, eficaz e realista.

Essa teoria não tem por objetivo medir os estilos de cada indivíduo nem tampouco rotulá-lo de forma estagnada, mas identificar o estilo de maior predominância na forma como cada um aprende e, com isso, elaborar o que é necessário desenvolver para estes indivíduos em relação aos outros estilos não predominantes. Esse processo deve ser realizado com base em um trabalho educativo que possibilite que os outros estilos também sejam contemplados na formação do aluno.

A predominância dos estilos de aprendizagem podem ou não modificar ao longo da vida do indivíduo, depende do ambiente e do trabalho em que o Sujeito está inserido. Os estilos são, portanto, flexíveis. Para identificar os estilos de aprendizagem, o instrumento que pode ser utilizado é o CHAEA - (Cuestionário Honey y Alonso de Estilos de Aprendizaje, disponível em: www.estilosdeaprendizaje.es). Esse modelo de questionário, que identifica os estilos aperfeiçoa e complementa os demais questionários, atualizando-os de acordo com as necessidades emergentes. O CHAEA é composto de oitenta itens no total, sendo vinte itens equivalentes a cada estilo, e também contempla uma série de perguntas sócio-acadêmicas que permitem relacionar variáveis de idade, gênero, número de anos de experiência, etc. 


\section{Revista Docência e Cibercultura}

A partir desses referenciais de aprendizagem e dos novos elementos do virtual, as investigações realizadas por Kerckhove (1999; 1995) e Lévy (1993; 1996), evidenciam informações sobre como o espaço virtual possibilita formas de aprendizagem diferenciadas das formas de aprendizagem tradicionais. Portanto, os estudos realizados sobre essa temática, juntamente com a teoria de estilos de aprendizagem facilitaram um perfil sobre como as pessoas utilizam o virtual e as formas de direcionar as aplicações didáticas e pedagógicas para o processo de ensino e aprendizagem.

De acordo com as pesquisas anteriormente desenvolvidas por Barros (2011; 2013), o tipo de aprendizagem que ocorre no espaço virtual se inicia por meio da busca de dados e informações, após um estímulo previamente planejado; em seguida a essa busca, ocorre a organização do material encontrado de forma particular, de acordo com as formas pessoais de elaboração, organização, análise e síntese. Por fim, a produção de uma aplicação multimídia com os instrumentos disponibilizados. Embasando-se nesses elementos norteadores do virtual para a educação e a teoria dos estilos de aprendizagem, Barros $(2011$; 2013) desenvolveu um instrumento de identificação do estilo de uso do espaço virtual.

Segundo a autora, os estilos de uso do espaço virtual podem ser entendidos como níveis de utilização dos aplicativos e ferramentas, interfaces online baseadas - entre outras características - na busca de informação, no planejamento e na imagem. Categorizou-se, neste trabalho, a existência de quatro tendências de uso do espaço virtual, apresentadas a seguir:

- estilo de uso participativo no espaço virtual: considera a participação como elemento central, no qual o indivíduo deve ter a ambiência do espaço. Além disso, para realizar um processo de aprendizagem no virtual, necessita de metodologias e materiais que priorizem o contato com grupos online,que solicite buscar situações online, realizar trabalhos em grupo, realizar fóruns de discussão e dar ações aos materiais desenvolvidos;

- estilo de uso busca e pesquisa no espaço virtual: tem como elemento central para a aprendizagem a necessidade de fazer pesquisa online, buscar informações de todos os tipos e formatos. Este estilo caracterizou-se como busca e pesquisa, no qual o usuário aprende mediante a busca,seleção e organização do conteúdo. Os materiais de aprendizagem devem estar voltados a construções e sínteses que englobem a pesquisa de um conteúdo; 


\section{Revista Docência e Cibercultura}

- estilo de estruturação e planejamento no espaço virtual: tem como elemento central para a aprendizagem a necessidade de desenvolver atividades que valorizem os aplicativos para elaborar conteúdos e atividades de planejamento.

- estilo de ação concreta e produção no espaço virtual: tem como elemento central para a aprendizagem a necessidade de realização dos serviços online e a rapidez na realização desse processo. Viabilizar com rapidez é um dos eixos centrais deste estilo de uso; utilizar o espaço virtual como um espaço de ação e produção.

\section{PROCEDIMENTOS METODOLÓGICOS}

\subsection{Desenho do estudo}

As opções metodológicas deste estudo basearam-se em uma diversidade de estratégias de recolha de informação, privilegiando, o recurso das metodologias qualitativas, tendo em conta o objeto do estudo. Não optamos por denominar o trabalho desenvolvido por estudo de caso que, para Yin (2011), trata-se de uma abordagem metodológica de investigação quando queremos compreender, explorar ou descrever acontecimentos e contextos complexos, nos quais estão simultaneamente envolvidos diversos fatores.

Porém, devido à dimensão do trabalho, que limita a introdução de elementos de informação complementares que poderiam conduzir a processos de triangulação de dados assim uma maior contextualização dos estudos, optou-se por designá-los apenas por estudo para a construção de estratégias didáticas e pedagógicas para promover o estudo dos estilos nos fóruns online. Tendo o presente estudo o objetivo apresentar diretrizes de como potencializar o trabalho pedagógico com os fóruns de discussão em contextos online, ampliando o conceito e a função pedagógica dos mesmos com a teoria dos estilos de aprendizagem. O contexto de estudo circunscreve cursos realizados em áreas de Educação, totalmente online de forma assíncrona, em plataforma online e com recursos abertos desenhados para os mesmos.

A realização do estudo aqui descrito teve como sustentação teórica as seguintes referências: Alonso, Gallego e Honey (2007), Barros (2009; 2011; 2013; 2015; 2016); Dias (2013), Pereira, et al (2007) e Santos (2013, 2014, 2016, 2017a, 2017b).

\subsection{Questões do estudo}




\section{Revista Docência e Cibercultura}

A partir da preocupação dos estudos dos fóruns e a dimensão e posição da sua importância para os processo de comunicação assíncrona, os fóruns nos últimos tempos tem tomado um papel central no trabalho de motivação, diálogo e tentativa de trabalho colaborativo da educação online (GRITSENKO, 2016). O aporte oferecido pelos estilos de aprendizagem e pelos estilos de uso do espaço virtual permite que as estratégias sejam revistas e até mesmo inovadas nos fóruns online. O questionamento central destaca: como os fóruns online podem ser potencializados com estratégias didático pedagógicas a partir da teoria de estilos de aprendizagem? Ademais, seguem os questionamentos secundários:

- Qual a nova dimensão didático pedagógica dos fóruns online no trabalho colaborativo?

- Como fazer dos fóruns um espaço de real comunicação assíncrona?

- Quais as diretrizes didático pedagógicas podemos considerar na construção dessa potencialização dos fóruns online?

\section{$5.3 \mathrm{O}$ contexto e amostra}

A Universidade Aberta, localizada em Lisboa (Portugal) é uma instituição de pequeno porte, mas que tem um modelo pedagógico premiado por ser o primeiro modelo de eLearning em língua portuguesa, a plataforma utilizada é o moodle 3.0 customizada para o modelo pedagógico da Universidade cujos 150 professores atendem cerca de 10.000 alunos em todos os países de língua portuguesa. Para fins deste estudo, a coleta de dados incluiu os registros de interação do fórum de duas disciplinas dos curso de pós-graduação e graduação na área da Educação. As duas disciplinas são ministradas nos segundos anos dos cursos.

O perfil dos alunos deste curso inclui as seguintes características: a maioria tem mais de 35 anos, trabalhadores e com filhos. A relação que estabelecem com a educação é muito clara e visa crescer na carreira profissional ou para que possam conseguir um outro emprego na área específica da pós-graduação. A amostra foi composta pelo total de 62 estudantes: 45 da graduação e 17 da pós-graduação.

Tabela 1 - Características da amostra

\begin{tabular}{|c|c|c|c|c|}
\hline ב Observados & Gênero & Idade & Estudantes & $\begin{array}{l}\text { Média de participação } \\
\text { por estudantes nos } \\
\text { fóruns }\end{array}$ \\
\hline
\end{tabular}

\begin{tabular}{l|l|l} 
v.2 & n.1 & p. 25
\end{tabular}




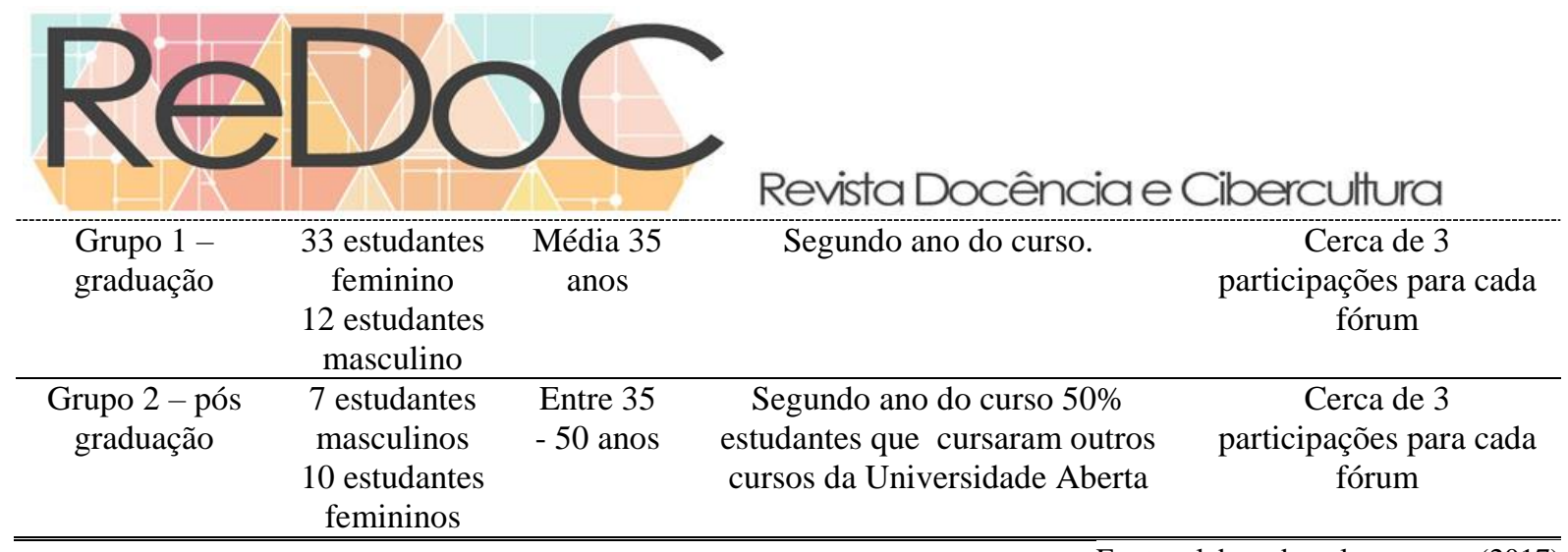

Fonte: elaborado pelas autoras (2017)

\subsection{A proposta inovadora e a forma de coleta de dados}

Foi desenhada uma proposta de estratégias para o desenvolvimento dos fóruns de acordo com as tendências de inovação e melhoria do potencial dos cursos online (RASMUSSEN, 2017; ROTHWELL, 2013). Essa proposta foi estruturada a partir dos estilos de uso do espaço virtual e as diretrizes para a construção de exercícios e atividades para o espaço virtual (BARROS, 2014; 2016). O tipo de trabalho realizado nos fóruns se configurou em dois tipos de estratégia, quais sejam: uma na diversificação de atividades e exercícios e a outra na diversificação da tipologia de perguntas. Os estilos de uso do espaço virtual dividem-se em 4 tipos e a partir de cada qual foram desenhadas as atividades e exercícios, bem como as perguntas. O objetivo era diversificar e potencializar os fóruns online na dimensão da interação e da participação pelos estudantes. A proposta foi organizada de acordo com as orientações que seguem no quadro 1:

\section{Quadro 1 - Estratégias para o uso dos Fóruns - curso de graduação e pós graduação Universidade Aberta ( Portugal)}

Estratégi No total foram 3 fóruns desenvolvidos durante cada uma das disciplinas. Aqui consideramos

a somente os fóruns de conteúdo dos temas. Cada fórum teve um tema específico e a duração de

didática duas a três semanas. A participação nos fóruns foi sempre considerada como quesito obrigatório tendo uma porcentagem na somatória da avaliação final.

Segue a estrutura de atividades/ perguntas para cada fórum dos três temas das Unidades Curriculares

Tema 1 - Graduação

A-busca e pesquisa online com orientações de como realizar a pesquisa, onde e com que objetivo.

B-síntese do tema com base em critérios pré definidos e orientados no fórum.

C- mapa conceitual do tema utilizando recursos da web 2.0 ou os aplicativos comuns como word ou ppt.

D-elaboração de um ppt ou prezi sobre o tema com base nas orientações técnicas disponibilizadas.

Tema 2 -Graduação

A-grupo de trabalho na resposta a uma pergunta relativa ao tema.

B-reflexão sobre uma pergunta central do tema.

C-organização de um quadro comparativo sobre o que sabia do tema e o que aprendeu de novo.

\begin{tabular}{l|l|l|l|l|l|l} 
(C) Redoc & Rio de Janeiro & v.2 & n.1 & p. 26 & Jan/Abr. 2018 & ISSN 2594-9004
\end{tabular}




\section{Revista Docência e Cibercultura}

D-realização de busca de imagens ou uma sequência de imagens relacionadas ao tema.

Tema 3 - Graduação

A-participação em uma wiki colaborativa sobre o tema em questão

B-análise crítica sobre uma afirmação central inserida no tema

C-estruturar uma tabela como as ideias principais referentes aos conceitos, características e história.

D-construir no google docs um texto síntese do tema em questão.

Tema 4 - Graduação

A-pesquisa sobre três links relacionados ao tema, disponibilização dos links e uma síntese do conteúdo do link e sua relação ao tema.

B- resumo das principias ideias sobre o tema em no máximo 20 linhas.

C-explicar o conceito, características e aplicabilidade do tema.

D-produção de um vídeo com aplicativos da web 2.0

Tema1 - pós-graduação

A -busca e pesquisa online com orientações de como realizar a pesquisa, onde e com que objetivo

B - síntese do tema com base em critérios pré definidos e orientados no fórum

C- mapa conceitual do tema utilizando recursos da web 2.0 ou os aplicativos comuns como word ou ppt.

D- elaboração de um ppt ou prezi sobre o tema com base nas orientações técnicas disponibilizadas

Tema 2 pós-graduação

A- grupo de trabalho na resposta a uma pergunta relativa ao tema.

B-reflexão sobre uma pergunta central do tema.

C- organização de um quadro comparativo sobre o que sabia do tema e o que aprendeu de novo.

D-elaboração de uma busca de imagens ou uma sequência de imagens relacionadas ao tema.

Tema 3 pós- graduação

A-participação em uma wiki colaborativa sobre o tema em questão

B-análise crítica sobre uma afirmação central inserida no tema.

C-apresentar em uma tabela as principais características sobre o tema divididos.

D-construir no google docs um texto síntese do tema em questão.

Tema 4 pós-graduação

A-pesquisa sobre três links relacionados ao tema, disponibilização dos links e uma síntese do conteúdo do link e sua relação ao tema.

B- resumo das principias ideias sobre o tema em no máximo 20 linhas

C- exemplificar um plano de projeto a partir das orientações do tema.

D-produção de um vídeo com aplicativos da web 2.0.

Critérios - Deveriam ter, no mínimo, 3 participações consubstanciadas;

de - Seguir uma netetiqueta, ou seja, escrever as mensagens cumprimentando os colegas,

avaliação desenvolvendo o assunto e finalizando com uma saudação;

- Os conteúdos e as análises críticas solicitadas deveriam ter por base os materiais e textos

indicados na disciplina e deveriam ser citados nas argumentações realizadas;

-As atividades solicitadas deveriam ser desenvolvidas plenamente de acordo com a escolha feita.

Interaçã Fóruns abertos e com orientações para a interação em todos os momentos de forma assíncrona e espontânea de acordo com as exigências para cada fórum.

Interação entre aluno - docente, correu de forma normal com as intervenções e o estímulo motivacional do docente para que as atividades, participações e exercícios fossem realizados.

\begin{tabular}{l|l|l|l|l|l|l} 
(C) Redoc & Rio de Janeiro & v.2 & n.1 & p. 27 & Jan/Abr. 2018 & ISSN 2594-9004
\end{tabular}




\section{Revista Docência e Cibercultura}

Interação entre aluno - aluno, ocorreu de forma mais intensiva nos posts e atividades propostas. Interação aluno- conteúdo, com as leituras e materiais disponibilizados sobre o tema proposto.

Estilos A partir dos estilos foram elaboradas as estratégias acima citadas. Os estilos de uso participativo de uso do no espaço virtual motivou as interações voltadas as atividades de grupo e participação espaço colaborativa.

virtual Já o estilo de uso busca e pesquisa no espaço virtual motivou as interações de busca e pesquisa online a partir de temas e desafios propostos.

O estilo de estruturação e planejamento no espaço virtual motivou a interação com uso de estruturas e esquemas como mapas conceituais e quadros comparativos ou de análise sobre os temas do conteúdo.

E por fim o estilo de ação concreta e produção no espaço virtual motivou a interação na produção em ppts, prezis ou vídeos com os tema solicitados.

Fonte: desenvolvido pelas autoras (2017)

Com esta proposta aplicada a cada unidade curricular, os dados recolhidos foram o número de participações em cada fórum e em cada atividade proposta. Sendo que os estudantes poderiam optar por uma ou todas das quatro possibilidades. Para tanto aplicamos os testes estatísticos no programa SPSS e os resultados seguem a seguir.

\section{RESULTADOS}

Para decidir os tipos de testes seriam utilizados, realizou-se o procedimento iniciail de estudos das amostra de acordo com a sequência descrita a seguir:

Tabela 1 - Quantidade total e distribuição por nível de escolaridade

\begin{tabular}{ccccc}
\hline & Frequência & Porcentagem & Porcentagem válida \\
\hline \multirow{2}{*}{ Válido } & graduação & 52 & 75,4 & 75,4 \\
\cline { 2 - 4 } & pós-graduação & 17 & 24,6 & 24,6 \\
\hline Total & 69 & 100,0 & 100,0 \\
\hline
\end{tabular}

Fonte: resultado obtido por meio de teste estatístico realizado no SPSS 25 pelas autoras

Os testes estão subdivididos em etapas que passamos a sequenciar e a descrever. 
Etapa 1 - Teste de normalidade para definição dos testes estatísticos subsequentes (paramétricos ou nãoparamétricos) Nossa opção pelo teste de Kolmogorov Smirnov se deu em função da quantidade de unidades de análise que é de 62. Recomenda-se o uso do teste de Shapiro Wilk apenas para amostras menores que 50 unidades de análise.

Etapa 2: A formulação das hipóteses foi realizada considerando a homogeneidade e a diferença das amostras.

H0: A distribuição da variável NÃO difere de uma distribuição normal (hipótese da homogeneidade)

H1: A distribuição da variável difere de uma distribuição normal (hipótese das diferenças)

Nível de significância $=5 \%-0,05$

Se p value for < que 0,05 , rechaçamos a hipótese nula.

Tabela 2 - Teste de Kolmogorov-Smirnov de uma amostra

\begin{tabular}{llr}
\hline & & \multicolumn{1}{c}{ Estudantes } \\
\hline $\mathrm{N}$ & & 69 \\
\hline Parâmetros normais & Média & 1,2464 \\
& Desvio Padrão &, 43406 \\
Diferenças Mais Extremas & Absoluto &, 468 \\
& Positivo &, 468 \\
Estatística do teste & Negativo &,- 285 \\
Significância Sig. (bilateral) & &, 468 \\
\hline
\end{tabular}

Fonte: resultado obtido por meio de teste estatístico realizado no SPSS 25 pelas autoras

Tabela 3 - Teste de normalidade para cada um dos grupos em uma mesma amostra

\begin{tabular}{|c|c|c|c|}
\hline Estudantes & & & lantes \\
\hline graduação & $\mathrm{N}$ & & 52 \\
\hline & Parâmetros normais & Média & 1,0000 \\
\hline & & Desvio Padrão &, $00000^{\mathrm{c}}$ \\
\hline pós-graduação & $\mathrm{N}$ & & 17 \\
\hline & Parâmetros normais & Média & 2,0000 \\
\hline
\end{tabular}




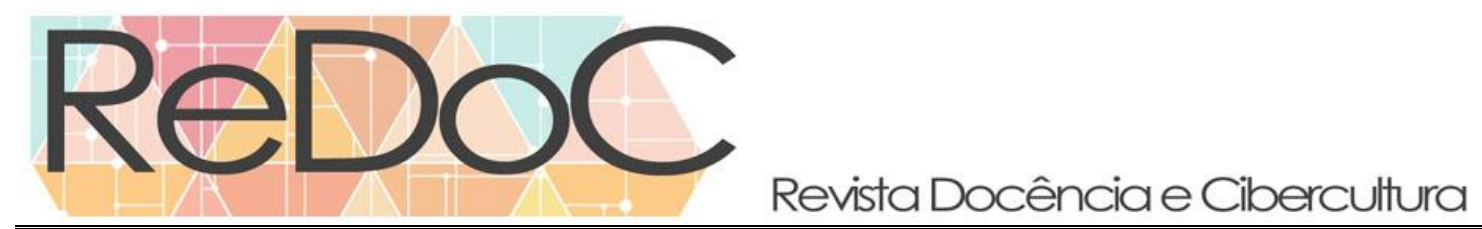

\section{Desvio Padrão \\ , $00000^{\mathrm{c}}$}

Fonte: resultado obtido por meio de teste estatístico realizado no SPSS 25 pelas autoras

As amostras não são normais, ou seja, a distribuição não é normal. Desta forma, os testes utilizados foram os não-paramétricos.

Etapa 3: Apresentação dos dados dos fóruns

Para efeito de comparação, dividimos os grupos entre alunos de graduação e alunos de pós-graduação. Os fóruns foram assim denominados: a letra $\mathrm{F}$ denomina o Fórum, seguido do número 1, 2, 3 ou 4 (quantidade de fóruns que analisados) e as letras denominam os estilos $\mathrm{A}$, $\mathrm{B}, \mathrm{C}$ e D.

Tabela 4 - Estatística de participação nos Fóruns de cada tema

\begin{tabular}{ccccc}
\hline \hline Estudantes & Fórum1 & Fórum 2 & Fórum3 & Fórum4 \\
\hline Graduação & $\begin{array}{c}\text { F1A - 52 } \\
\text { participações }\end{array}$ & $\begin{array}{c}\text { F2A-57 } \\
\text { participações }\end{array}$ & $\begin{array}{c}\text { F3A - 77 } \\
\text { participações }\end{array}$ & $\begin{array}{c}\text { F4A - 46 } \\
\text { participações }\end{array}$ \\
Pós-graduação & F 1 B e F 1 D- & $\begin{array}{c}\text { F2A - 36 } \\
\text { participações }\end{array}$ & $\begin{array}{c}\text { F3A - 31 } \\
\text { participações }\end{array}$ & $\begin{array}{c}\text { F4A 16 } \\
\text { participações }\end{array}$ \\
17 estudantes & $\begin{array}{c}\text { participações m } \\
\text { em cada um }\end{array}$ & & & \\
\hline
\end{tabular}

Fonte: resultado obtido por meio de teste estatístico realizado no SPSS 25 pelas autoras

Os resultados apresentados destacam que a participação dos estudantes tanto da graduação como da pós graduação foi centrada na atividade 1 de todos os fóruns. Também pode-se perceber que na maioria dos fóruns a participação foi mais que uma vez. As estratégias da atividade 1 foram todas centradas no estilo ativo de participação do uso do virtual Barros (2011; 2013). Isso significa que a realização das mesmas tem uma tendência e caracterização do tipo de uso que as pessoas realizam no espaço online, ou seja, atividades que consideram a participação como elemento central, o contato com grupos online, a busca de situações online e realização de trabalhos em grupo. Isso nos ajuda a compreender que os fóruns realmente 


\section{Revista Docência e Cibercultura}

precisam serem movimentados com atividades assertivas que facilitem essa participação com os conteúdos e com as pessoas.

\section{Etapa 4: Teste de hipótese para cada fórum e por estilo}

Para efeito de comparação, dividimos os grupos entre alunos de graduação e alunos de pós-graduação. Os fóruns foram assim denominados: a letra $\mathrm{F}$ denomina o Fórum, seguido do número 1, 2, 3 ou 4 (quantidade de fóruns que analisados) e as letras denominam os estilos $\mathrm{A}$, B, C e D. Para os estudantes de graduação as hipóteses que se colocam são:

H0: A participação dos alunos NÃO é diferente nos fóruns (hipótese da homogeneidade)

H1 : A participação dos alunos é diferente nos fóruns(hipótese das diferenças)

\begin{tabular}{|c|c|c|c|}
\hline \multicolumn{4}{|c|}{ Resumo de Teste de Hipótese } \\
\hline & Hipótese nula & Sig. & Decisão \\
\hline 1 & $\begin{array}{ll}\text { Teste de } \\
\text { A sequência de valores definida pớxecuçôes } \\
\text { F1A e e é aleatória. } & \text { de uma } \\
\text { amostra }\end{array}$ & & $\begin{array}{l}\text { Incapaz } \\
\text { de } \\
\text { calcular. }\end{array}$ \\
\hline 2 & 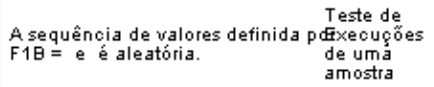 & & $\begin{array}{l}\text { Incapaz } \\
\text { de } \\
\text { calcular. }\end{array}$ \\
\hline 3 & $\begin{array}{l}\text { A sequência de valores definida pof́xecuçốes } \\
\begin{array}{ll}\text { de uma } \\
\text { amo e é aleatória. } & \text { amostra }\end{array}\end{array}$ & & $\begin{array}{l}\text { Incapaz } \\
\text { de } \\
\text { calcular. }\end{array}$ \\
\hline 4 & $\begin{array}{l}\text { A sequência de valores definida pớxecuçốes } \\
\begin{array}{ll}\text { de uma } \\
\text { F1D }=(1) \text { e (0) é aleatória. } & \text { amostra }\end{array}\end{array}$ & .079 & $\begin{array}{l}\text { Reter a } \\
\text { hipótese } \\
\text { nula. }\end{array}$ \\
\hline 5 & $\begin{array}{ll}\text { Aeste de } \\
\text { A sequência de valores definida pớxecuốes } \\
\text { F2A = é aleatória. } & \text { de uma } \\
\text { amostra }\end{array}$ & & $\begin{array}{l}\text { Incapaz } \\
\text { de } \\
\text { calcular. }\end{array}$ \\
\hline 6 & 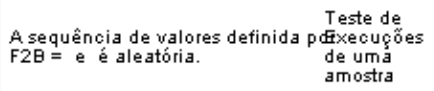 & & $\begin{array}{l}\text { Incapaz } \\
\text { de } \\
\text { calcular. }\end{array}$ \\
\hline 7 &  & & $\begin{array}{l}\text { Incapaz } \\
\text { de } \\
\text { calcular. }\end{array}$ \\
\hline 8 & 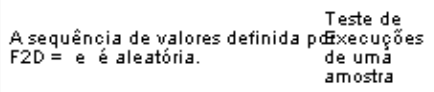 & & $\begin{array}{l}\text { Incapaz } \\
\text { de } \\
\text { calcular. }\end{array}$ \\
\hline 9 & $\begin{array}{ll}\text { A sequência de valores definida pớxecuçốes } \\
\text { F3A= e é aleatória. } & \text { de uma } \\
\text { amostra }\end{array}$ & & $\begin{array}{l}\text { Incapaz } \\
\text { de } \\
\text { calcular. }\end{array}$ \\
\hline 10 & $\begin{array}{l}\text { Teste de } \\
\begin{array}{ll}\text { A sequência de valores definida pợecuốos } \\
\text { F } 3 \mathrm{~B}=\text { e é aleatória. } & \text { de uma } \\
\text { amostra }\end{array}\end{array}$ & & $\begin{array}{l}\text { Incapaz } \\
\text { de } \\
\text { calcular. }\end{array}$ \\
\hline 11 & $\begin{array}{l}\text { Aeste de } \\
\begin{array}{ll}\text { A sequência de valores definida pớxecuçónes } \\
\text { de uma é aleatória. } & \text { amostra }\end{array}\end{array}$ & & $\begin{array}{l}\text { Incapaz } \\
\text { de } \\
\text { calcular. }\end{array}$ \\
\hline
\end{tabular}

Resumo de Teste de Hipótese

\begin{tabular}{|c|c|c|c|c|}
\hline & Hipótese nula & Teste & Sig. & Decisão \\
\hline 12 & $\begin{array}{l}\text { A sequência de valores definida po } \\
F 3 D=e \text { é aleatória. }\end{array}$ & $\begin{array}{l}\text { Teste de } \\
\text { pofxecuçốes } \\
\text { de uma } \\
\text { amostra }\end{array}$ & & $\begin{array}{l}\text { Incapaz de } \\
\text { calcular. }\end{array}$ \\
\hline 13 & $\begin{array}{l}\text { A sequência de valores definida po } \\
\text { F4A = é aleatória. }\end{array}$ & $\begin{array}{l}\text { Teste de } \\
\text { pdixecuçốes } \\
\text { de uma } \\
\text { amostra }\end{array}$ & . & $\begin{array}{l}\text { Incapaz de } \\
\text { calcular. }\end{array}$ \\
\hline 14 & $\begin{array}{l}\text { A sequência de valores definida } \mathrm{p} \\
\mathrm{F} 4 \mathrm{~B}=\text { e é aleatória. }\end{array}$ & $\begin{array}{l}\text { Teste de } \\
\text { pdixecuçốes } \\
\text { de uma } \\
\text { amostra }\end{array}$ & & $\begin{array}{l}\text { Incapaz de } \\
\text { calcular. }\end{array}$ \\
\hline 15 & $\begin{array}{l}\text { A sequência de valores definida } \mathrm{p} \\
\mathrm{F} 4 \mathrm{C}=\text { e é aleatória. }\end{array}$ & $\begin{array}{l}\text { Teste de } \\
\text { pdixecuçốes } \\
\text { de uma } \\
\text { amostra }\end{array}$ & . & $\begin{array}{l}\text { Incapaz de } \\
\text { calcular. }\end{array}$ \\
\hline 16 & $\begin{array}{l}\text { A sequência de valores definida po } \\
F 4 D=(0) \text { e (1) é aleatória. }\end{array}$ & $\begin{array}{l}\text { Teste de } \\
\text { pdixecuçốes } \\
\text { de uma } \\
\text { amostra }\end{array}$ & .000 & $\begin{array}{l}\text { Rejeitar a } \\
\text { hipótese } \\
\text { nula. }\end{array}$ \\
\hline
\end{tabular}

São exibidas significâncias assintótic as. O nivel de significância é ,05. 
Os resultados destacam que a participação dos alunos se tornou diferente à medida que os fóruns foram evoluindo (provavelmente em função do desenvolvimento do costumo e do hábito), de modo que a participação de tipo D no Fórum 4 tornou-se mais evidente (comprovado pelo teste estatístico apresentado).

Para os estudantes de pós-graduação as hipóteses que se colocam são:

H0: A participação dos alunos NÃO é diferente nos fóruns (hipótese da homogeneidade)

H1: A participação dos alunos é diferente nos fóruns(hipótese das diferenças)

Resumo de Teste de Hipótese

\begin{tabular}{|c|c|c|c|}
\hline & Hipótese nula & Sig. & Decisão \\
\hline 1 & $\begin{array}{lc}\text { Teste de } \\
\text { A sequência de valores definida pofixecuçốes } \\
\text { de uma } \\
\text { amostra }\end{array}$ & & $\begin{array}{l}\text { Incapaz } \\
\text { de } \\
\text { calcular. }\end{array}$ \\
\hline 2 & $\begin{array}{lc}\text { Teste de } \\
\text { Asequência de valores definida pofxecuçốes } \\
\text { F1日 e é aleatória. } & \text { de uma } \\
\text { amostra }\end{array}$ & & $\begin{array}{l}\text { Incapaz } \\
\text { de } \\
\text { calcular. }\end{array}$ \\
\hline 3 & $\begin{array}{lc}\text { Teste de } \\
\text { A sequência de valores definida pofxecuçốes } \\
\text { F1C = (1) e (0) é aleatória. } & \text { de uma } \\
\text { amostra }\end{array}$ & .605 & $\begin{array}{l}\text { Reter a } \\
\text { hipótese } \\
\text { nula. }\end{array}$ \\
\hline 4 & $\begin{array}{lc}\text { Teste de } \\
\begin{array}{ll}\text { A sequência de valores definida pof́xecuçóes } \\
\text { F1D }=(1) \text { e (0) é aleatória. } & \text { de uma } \\
\text { amostra }\end{array}\end{array}$ & .787 & $\begin{array}{l}\text { Reter a } \\
\text { hipótese } \\
\text { nula. }\end{array}$ \\
\hline 5 & $\begin{array}{lc}\text { Teste de } \\
\text { A sequência de valores definida pof́xecuçốes } \\
\text { de uma } \\
\text { amostra }\end{array}$ & . & $\begin{array}{l}\text { Incapaz } \\
\text { de } \\
\text { calcular. }\end{array}$ \\
\hline 6 & $\begin{array}{lc}\text { Teste de } \\
\text { A sequência de valores definida pof́xecuçốes } \\
\text { F2B e é aleatória. } & \text { de uma } \\
\text { amostra }\end{array}$ & & $\begin{array}{l}\text { Incapaz } \\
\text { de } \\
\text { calcular. }\end{array}$ \\
\hline 7 & $\begin{array}{lc}\text { Aeste de } \\
\text { A sequência de valores definida pof́xecuçốes } \\
\text { de uma e é aleatória. } \\
\text { amostra }\end{array}$ & . & $\begin{array}{l}\text { Incapaz } \\
\text { de } \\
\text { calcular. }\end{array}$ \\
\hline 8 & $\begin{array}{lc}\text { A sequência de valores definida pof́xecuçốes } \\
\text { de uma } \\
\text { F2D e é aleatória. }\end{array}$ & . & $\begin{array}{l}\text { Incapaz } \\
\text { de } \\
\text { calcular. }\end{array}$ \\
\hline 9 & $\begin{array}{lc}\text { Aeste de } \\
\text { A sequência de valores definida pofxecuçốes } \\
\text { fa uma e é aleatória. } & \text { amostra }\end{array}$ & . & $\begin{array}{l}\text { Incapaz } \\
\text { de } \\
\text { calcular. }\end{array}$ \\
\hline 10 & $\begin{array}{lc}\text { A sequência de valores definida pofixecuçốes } \\
\text { F3B }=(0) \text { e (1) é aleatória. } & \begin{array}{c}\text { de uma } \\
\text { amostra }\end{array}\end{array}$ & .884 & $\begin{array}{l}\text { Reter a } \\
\text { hipótese } \\
\text { nula. }\end{array}$ \\
\hline 11 & $\begin{array}{lc}\text { Teste de } \\
\text { A sequência de valores definida pofixecuçốes } \\
\mathrm{F} 3 \mathrm{C}=(1) \text { e (0) é aleatória. } & \text { de uma } \\
\text { amostra }\end{array}$ & .626 & $\begin{array}{l}\text { Reter a } \\
\text { hipótese } \\
\text { nula. }\end{array}$ \\
\hline
\end{tabular}

São exibidas signific âncias assintótic as. O nível de significância é ,05. 


\begin{tabular}{|c|c|c|c|c|}
\hline \multicolumn{5}{|c|}{ Resumo de Teste de Hipótese } \\
\hline & Hipótese nula & Teste & Sig. & Decisão \\
\hline 12 & $\begin{array}{l}\text { A sequência de valores definida } p \\
F 3 D=(1) \text { e (0) é aleatória. }\end{array}$ & $\begin{array}{l}\text { Teste de } \\
\text { dixecuçốes } \\
\text { de uma } \\
\text { amostra }\end{array}$ & .891 & $\begin{array}{l}\text { Reter a } \\
\text { hipótese } \\
\text { nula. }\end{array}$ \\
\hline 13 & $\begin{array}{l}\text { A sequência de valores definida } \\
\mathrm{F} 4 \mathrm{~A}=\mathrm{e} \text { é aleatória. }\end{array}$ & $\begin{array}{l}\text { Teste de } \\
\text { dixecuçốes } \\
\text { de uma } \\
\text { amostra }\end{array}$ & . & $\begin{array}{l}\text { Incapaz } \\
\text { de } \\
\text { calcular. }\end{array}$ \\
\hline 14 & $\begin{array}{l}\text { A sequência de valores definida } \mathrm{p} \\
\mathrm{F} 4 \mathrm{~B}=\text { e é aleatória. }\end{array}$ & $\begin{array}{l}\text { Teste de } \\
\text { dixecuçốes } \\
\text { de uma } \\
\text { amostra }\end{array}$ & & $\begin{array}{l}\text { Incapaz } \\
\text { de } \\
\text { calcular. }\end{array}$ \\
\hline 15 & $\begin{array}{l}\text { A sequência de valores definida } \mathrm{p} \\
\mathrm{F} 4 \mathrm{C}=\text { e é ale atória. }\end{array}$ & $\begin{array}{l}\text { Teste de } \\
\text { dixecuçốes } \\
\text { de uma } \\
\text { amostra }\end{array}$ & . & $\begin{array}{l}\text { Incapaz } \\
\text { de } \\
\text { calcular. }\end{array}$ \\
\hline 16 & $\begin{array}{l}\text { A sequência de valores definida } \\
\text { F4D }=\text { e é aleatória. }\end{array}$ & $\begin{array}{l}\text { Teste de } \\
\text { dfxecuçốes } \\
\text { de uma } \\
\text { amostra }\end{array}$ & & $\begin{array}{l}\text { Incapaz } \\
\text { de } \\
\text { calcular. }\end{array}$ \\
\hline
\end{tabular}

São exibidas significâncias assintóticas. O nível de significância é ,05.

Os resultados destacam que a participação dos alunos de pós-graduação ficou estabilizada - tendendo à homogeneização - em (todos os campos em que se afirma que se deve reter a hipótese nula). Com os testes, foi possível observar que as atividades em geral foram desenvolvidas com o aumento da participação dos estudantes na graduação e a interação dos mesmos. Este aspecto é um aspecto importantíssimo para o trabalho a ser desenvolvido nos fóruns. O movimento de interação proporcionado por essas estratégias facilitou inclusive o trabalho mais colaborativo pelas atividades foram organizadas. Dois aspectos podem ser entendidos, o tipo de atividades realizada por grupo ou a própria interação opinativa sobre o que o colega desenvolveu.

\section{CONSIDERAÇÕES FINAIS}

Os resultados obtidos, nos facilitam pensar que as estratégias didáticas a partir da teoria dos estilos de aprendizagem podem potencializar o trabalho nos fóruns online. Essas estratégias que apresentamos trazem diretrizes que podem ajudar na construção das mesmas. Essas diretrizes resumem-se em: utilizar os estilos de uso do espaço virtual para direcionar o tipo de atividade ou pergunta a ser elaborada para os fóruns. A função pedagógica dos fóruns pode ser ampliada a partir dessas diretrizes, não só pela comunicação que é a sua definição e característica central, mas também a construção de conhecimentos entre pares e o

\begin{tabular}{l|l|l|l} 
v.2 & n.1 & p. 33
\end{tabular}




\section{Revista Docência e Cibercultura}

desenvolvimento de atividades colaborativas. A proposta está em dinamizar os fóruns com uma aplicação pedagógica no design de construção para cada temática em questão nas disciplinas.

Em termos gerais, consideramos que a interpretação dos resultados obtidos possibilitaram entender que houve uma participação evolutiva e em outro uma participação mais estável, mas ambos os grupos apresentam essa participação. Para além disso foi possível perceber o tipo de estratégia mais atrativa sendo a vantagem de estar dinamizada para o trabalho colaborativo e até em rede, dinamizando a interação opinativa e dialógica entre os estudantes.

Finalmente, é importante ressaltar que este estudo é parte de um projeto maior em desenvolvimento sobre a didática online que amplia e aprofunda o paradigma do virtual nas estratégias didáticas e pedagógicas para o espaço online. No que antes foi referido resulta a necessidade de prosseguir e aprofundar este tipo de análise através de novos estudos empíricos, em paralelo com a necessidade de não descuidar o desenvolvimento do referencial teórico.

\section{REFERÊNCIAS}

ALONSO, C. M.; GALLEGO, D. J.; HONEY, P. Los estilos de aprendizaje: procedimientos de diagnóstico y mejora. Madrid: Mensajero, 2007.

BARROS, D. M. V. Reflexões de base para a Educação a Distância: o virtual como novo espaço educativo. In Revista UDESC Virtual, v.1, 2008.

BARROS, D. M. V. Estilos de uso do espaço virtual: como se aprende e se ensina no virtual? Revista Inter Ação. 34(1), 2009.

BARROS, D. M.V. Estilos de Aprendizagem e o uso das tecnologias. Santo Tirso: deFacto, Portugal, 2013.

BARROS, D. M.V.; MIRANDA, L; MORAIS, C. Estilos de aprendizagem de futuros professores e estratégias de ensino da matemática no 1.o ciclo do ensino básico in: BARROS, D. M.V. Estilos de aprendizagem na atualidade. Volume 1, 2011.

BARROS, D.M.V. Estilos de Aprendizagem e Tecnologias in. RAMAL, A.; SANTOS, E. ( org.). Mídias e tecnologias na educação presencial e a distância - 1. ed. - Rio de Janeiro: LTC, 2016. 


\section{Revista Docência e Cibercultura}

BARROS, D.M.V. Estilos de Aprendizaje y las Tecnologías: Medios didácticos en lo virtual. Editorial Académica Española, Madrid, 2012.

BARROS, D.M.V. Estilos de Coaprendizagem e alguns indicadores das Competências Digitais In Educación, Volumen XXIII n ${ }^{\circ} 45$, septiembre de 2014. Fondo Editorial: Universidad Católica del Peru, 2014.

BARROS, S. (Org.). Las TIC en el sistema universitario español. Madrid, CRUE, 2004.

BARROS; D. M. V. Estilo de aprendizagem colaborativo para o e-learning. Revista Linhas, UDESC, v. 12, n. 2, 2011.

BARROS; D. M. V MIRANDA; L. GOULÃO, M.F.; HENRIQUES, S.; MORAIS, C. (2012). Estilos de Coaprendizagem para uma coletividade aberta de pesquisa in: OKADA, A.(org.) Recursos Educacionais Abertos e Redes Sociais: coaprendizagem e desenvolvimento profissional. Colearn. Disponível em: http://oer.kmi.open.ac.uk/?page_id=387 Acesso em 14 out. 2017.

BIASUTTI, M.A comparative analysis of forums and wikis as tools for online collaborative learning, Computers \& Education August,vol 111, 158-171, Elsevier Ltd, 2017.

CHANG,Y.F; HSU,C.L; CHENY.R. Effetive use of online vídeo cases in a techology course for pre-service teachers. In: Zhang, Y. (ed.) Proceedings of 2011 3rd Internacional Conference on Education Technology and Computer, vol3, pp.49-53, IACSIT Changchun, 2011.

DIAS, P. Inovação pedagógica para a sustentabilidade da educação aberta e em rede. Educação, Formação \& Tecnologias, 2013. 6 (2), 4-14. Disponível em: http://eft.educom.pt Acesso em 07 out. 2017.

FREIRE, Paulo. Extensão ou comunicação? Prefácio de Jacques Chonchol $7^{\mathrm{a}}$ ed. Rio de Janeiro, Paz e Terra, 1983.

GALLEGO, D.J.; ALONSO, C.M. \& BARROS, D. M. V. Estilos de Aprendizaje desafíos para una educación inclusiva e innovadora. Coleção Estudos Pedagógicos. Whitebooks, Santo Tirso. Portugal, 2015. 
GARRISON, D.R.; CLEVELAND-INNES, M. Facilitating cognitive presence in online learning: Interaction is not enough. American Journal of Distance Education 19(3): 133-148, 2005 .

GERLOCK,J.A.; MCBRIDE, D.L. Managing Online Discussion Forums: Building Community by Avoiding the Drama Triangle. In: College Teaching: Taylor y Francis Online, Vol 61, 2013.

GOULÃO, M.F. Ensino Aberto a Distância: Cognição e Afectividade. Tese de Doutoramento em Ciências da Educação, na Especialidade de Formação de Adultos, Universidade Aberta, 2002.

GRITSENKO, V. Interaction on Online Forums and Group Communication: A Case Study of an IT Support Community. Procedia - Social and Behavioral Sciences. Volume 236, 14 December 2016, Pages 14-24. International Conference on Communication in Multicultural Society, 2016.

HABERMAS, J. Consciência moral e agir comunicativo. 2ed. Tradução de Guido A. De Almeida. Rio de Janeiro, Tempo Brasileiro, 2003.

HENRI, F.; K. LUNDGREN-CAYROL. Apprentissage collaboratif à distance: Pour comprendre et concevoir les environnements d'apprentissage virtuels, Québec, Presses de l’Université du Québec, 2001.

KERCKHOVE, D. A pele da cultura. Lisboa: Relógio D’agua, 1995.

KERCKHOVE, D. Inteligencias en conexión: hacia una sociedad de la Web. Barcelona: Gedisa, 1999.

KOLB, D.A.\& SMITH, S. User's guide for the learning-style inventory: A manual for teachers and trainers. Boston, TRGHayGroup, 1996.

LÉVY, P. As tecnologias da inteligência: o futuro do pensamento na era da informática. Rio de Janeiro: Editora 34, 1993.

LÉVY, P. O que é virtual? São Paulo: ed. 34, 1996.

LINARD M. Conception de dispositif et changement de paradigme en formation, Education permanente: 152/2002. 


\section{Revista Docência e Cibercultura}

LINARD M. Des machines et des hommes. Éditions universitaires, Paris 1990.

LOIDE,R.S.; MACIEL.C.;PEREIRA, C.V. Fóruns de discussão: análise de artigos apresentados no SBIE e no ESUD In ESUD XI Congresso Brasileiro de Ensino Superior a Distância Florianópolis / SC, agosto de 2014. Disponível em: http://esud2014.nute.ufsc.br/anais-esud2014/files/pdf/127404.pdf Acesso em 07 out 2017.

MARCONDES FILHO, Ciro. Princípio da razão durante, vol.III, tomo 5, "O conceito de comunicação e a epistemologia metapórica". São Paulo, Paulus, 2010.

MELLO, D. E \& BARROS, D.M.V. (2014) Didática no eLearning: aspectos teóricos e comunicacionais. TIC EDUCA 2014. 14 a 16 de novembro de 2014, Instituto da Educação da Universidade de Lisboa, 2014. Disponível em: http://ticeduca2014.ie.ul.pt Acesso em 14 out 2017.

MELlO, D.E.; BARROS, D. M.V. Didática do Online, 2014. Disponível em: https://repositorioaberto.uab.pt/ Acesso em 14 out 2017.

MOORE, M. Theory of transactional distance. In KEEGAN, D. Theoretical Principles of Distance Education, Routledge , 1997.

MOHAMAD, M.; SHAHARUDDIN, S. Online Forum Discussion to Promote Sense of Learning Community among the Group Members. International Education Studies; Vol. 7, No. $13 ; 2014$.

MOKOENA, S. Student teachers' experiences of teaching practice at open and distance learning institution in South Africa. Turkish Online Journal of Distance Education-TOJDE. Volume: 18 Number: 2 Article 10. April 2017.

PALlOFF, Rena; PRATT, Keith. Construindo Comunidades de Aprendizagem no Ciberespaço.Porto Alegre, Artmed, 2002.

PEREIRA, A.; QUINTAS MENDES, A.; MORGADO, L.; AMANTE, L.; BIDARRA, J. Modelo Pedagógico Virtual da Universidade Aberta, Lisboa, Universidade Aberta, 2007.

PEREIRA, Eva Waisros; MORAES, Raquel de Almeida. História da educação a distância e os desafios na formação de professores no Brasil. In: SOUZA, A. M.; FIORENTINI, L. M. R.; RODRIGUES, M. A. M. (Orgs.). Educação superior a distância: comunidade de trabalho e 


\section{Revista Docência e Cibercultura}

aprendizagem em rede (CTAR). Brasília: Universidade de Brasília, Faculdade de Educação, 2009.

PETERS, Otto. A educação a distância em transição: tendências e desafios. Trad. Leila Ferreira de Souza Mendes. São Leopoldo, RS: Ed. Unisinos, 2004.

RASMUSSEN, Karsten Boye. Data quality in online environments. In.: FIELDING, N.G. ; LEE, R. M.; BLANK, G. The Sage Handbook of Online Research Methods. 2. edition. Sage Publications, 2017.

ROTHWELL, J.D. In mixed company: communicating in small groups and teams (8th ed.). Boston, MA: Waldsworth, 2013.

SANTOS, V. M. dos. Materiais audiovisuais para aeducação a distância: a contribuição dos estilos de aprendizagem. Tese. (Doutorado em Educação Escolar) - Universidade Estadual Paulista "Júlio de MesquitaFilho", Faculdade de Ciências e Letras, Campus de Araraquara, 2013.

SANTOS, V. M. dos. Em busca do sensível: repensar os audiovisuais em uma proposta metapórica. Tese. (Doutorado em Meios e Processos Audiovisuais) - Universidade de São Paulo (USP), Escola de Comunicações e Artes (ECA), São Paulo - SP, 2017a.

SANTOS, V. M. dos. Comunicação como ruptura: nuances de uma nova teoria brasileira. Revista Intexto, no prelo, $2017 \mathrm{~b}$.

SANTOS, V. M.. Informational leading role: creating new audiovisual narratives with Movie Maker.In: CORREAA, Francisco Tupy Gomes (Org.). MIE EXPERT: Changing the reality of the world teaching with technology. 1ed. São Paulo: InMod, 2016.

SANTOS, V. M. Audiovisuais para a educação a distância: pensando as preferências por meio dos estilos de aprendizagem. Journal of Learning Styles, v. 7, p. 109-135, n. 2014.

TRACTEMBERG, L. Colaboração Docente e Ensino Colaborativo na Educação Superior em Ciências, Matemática e Saúde - Contexto, Fundamentos e Revisão Sistemática.Tese de Doutoramento, Universidade Federal do Rio de Janeiro, 2011.

YIN, R. K. Applications of Case Study Research. SAGE Publications, Inc; Third Edition, 2011. 